\title{
BMI as criterion to start the work-up in obesity
}

\section{Giorgio Radetti ${ }^{1}$, Antonio Fanolla ${ }^{2}$, Fiorenzo Lupi ${ }^{3}$, Alessandro Sartorio ${ }^{4}$ and Graziano Grugni ${ }^{4}$}

${ }^{1}$ Marienklinik, Bolzano, Italy, ${ }^{2}$ Observatory for Health Provincial Government, Bolzano, South Tyrol, Italy, ${ }^{3}$ Department of Pediatrics, Regional Hospital of Bolzano, Bolzano, Italy, and ${ }^{4}$ Istituto Auxologico Italiano, IRCCS, Experimental Laboratory for Auxo-endocrinological Research \& Division of Auxology, Piancavallo, Italy

We read with great interest the recent review by Pasquali et al. (1) concerning the approach to be used for the early recognition and treatment of the metabolic and endocrinological alterations often observed in patients with obesity.

One of the main points is to define which level of obesity should be considered to start the investigations aimed to identify the possible primary or secondary metabolic and/or endocrine changes. The need to balance high cost and inconvenience to patients undergoing these investigations on one hand and the need to promptly detect the level of obesity triggering the development of metabolic syndrome (MetS) on the other hand are the reasons for the aforementioned considerations.

For pediatricians, the presence of normal or even increased growth rate and bone age, as frequently seen in children with obesity, is valuable in absolutely excluding conditions such as Cushing disease, GH deficiency, and hypothyroidism. By contrast, such evaluation in adult patients with obesity should instead rely on other indices, as correctly underlined by Pasquali et al. Among these, the most widely used in clinical practice is BMI which, however, does not discriminate fat mass from lean mass. Since it is well known that the amount of fat mass and its distribution are the most important metabolic risk factors, other indices have been developed subsequently, which also incorporate these parameters. However, the European Society of Endocrinology Clinical Practice Guideline suggested that currently there are no data showing that more sophisticated methods are more useful than BMI to guide the endocrine work-up in obesity (1).

In this context, we have recently compared these indices for their ability to identify MetS in 1332 children and adolescents with obesity (778 females, 554 males; age $14.4 \pm 1.8$ years), including BMI, the Fat Mass Index (FMI), the Tri-Ponderal Mass Index (TMI), the Waist Circumference (WC), the Waist-to-Height ratio (WHtR), the Fat-Free Mass Index (FFMI), plus a new one, the Body Mass Fat Index (BMFI), which also adjusts BMI for body composition and for WC (2). The main outcome of our study was that BMI, which neither considers body composition nor fat distribution, performed as well as the other indices.

Furthermore, we have verified whether these results could be replicated also in an adult population with Prader-Willi syndrome (PWS) (3), the most common form of syndromic obesity, which appears to feature a more favorable metabolic profile in comparison to BMImatched people with common obesity, characterized by lower insulin resistance due to the preferential s.c. fat distribution (4). However, many subjects with PWS develop MetS over the years and this condition might be one of the risk factors responsible for excessive mortality in this pathological condition (5). Therefore, the possibility to promptly recognize MetS or the risk of MetS in people with PWS would be valuable in permitting implementation of more intensive primary prevention strategies in such patients. In fact, we obtained similar results to those in the previous paper in children/adolescents with essential obesity, showing that no indices outperformed the others.

In conclusion we strongly agree that, from the practical point of view, BMI should be considered the most suitable screening index to be used, mainly because of the ease of calculation and cost efficiency.

\section{Declaration of interest}

The authors declare that there is no conflict of interest that could be perceived as prejudicing the impartiality of this letter.

Published by Bioscientifica Ltd. 


\section{Funding}

This research did not receive any specific grant from any funding agency in the public, commercial or not-for-profit sector.

\section{References}

1 Pasquali R, Casanueva F, Haluzik M, van Hulsteijn L, Ledoux S, Monteiro MP, Salvador J, Santini F, Toplak H \& Dekkers OM. European Society of Endocrinology Clinical Practice Guideline: endocrine work-up in obesity. European Journal of Endocrinology 2020 182 G1-G32. (https://doi.org/10.1530/EJE-19-0893)

2 Radetti G, Fanolla A, Grugni G, Lupi F \& Sartorio A. Indexes of adiposity and body composition in the prediction of metabolic syndrome in obese children and adolescents: which is the best?
Nutrition, Metabolism, and Cardiovascular Diseases 201929 1189-1196. (https://doi.org/10.1016/j.numecd.2019.06.011)

3 Radetti G, Fanolla A, Lupi F, Sartorio A \& Grugni G. Accuracy of different indexes of body composition and adiposity in identifying metabolic syndrome in adult subjects with Prader-Willi syndrome. Journal of Clinical Medicine 20209 E1646. (https://doi.org/10.3390/ jcm9061646)

4 Theodoro MF, Talebizadeh Z \& Butler MG. Body composition and fatness patterns in Prader-Willi syndrome: comparison with simple obesity. Obesity 200614 1685-1690. (https://doi.org/10.1038/ oby.2006.193)

5 Grugni G, Crinò A, Bedogni G, Cappa M, Sartorio A, Corrias A, Di Candia S, Gargantini L, Iughetti L, Pagano C et al. Metabolic syndrome in adult patients with Prader-Willi syndrome. Nutrition, Metabolism, and Cardiovascular Diseases 201323 1134-1140. (https:// doi.org/10.1016/j.numecd.2012.11.006)

Received 9 June 2020

Revised version received 23 June 2020

Accepted 20 July 2020 